\title{
Mielopatia de causa rara e tratável: relato de caso
}

Joana Rita Mendes, ${ }^{1}$ Ana Filipa Miranda, ${ }^{2}$ Maria Pieri Moreira ${ }^{3}$

\section{RESUMO}

Introdução: As fístulas arteriovenosas durais espinhais (FAVDE) são raras e a sua apresentação clínica é inespecífica e insidiosa. A etiologia é desconhecida e tem incidência superior em homens de meia-idade.

Descrição do Caso: Mulher de 50 anos, com antecedentes de depressão reativa, que se dirigiu ao médico de família por queixas álgicas após queda da própria altura com impacto na anca direita e joelhos. $\mathrm{O}$ exame objetivo e as imagens radiográficas não revelaram alterações importantes; contudo, as queixas persistiram apesar do tratamento conservador. O quadro inicial de coxalgia direita com predomínio noturno acresceu-se de disestesias dos membros inferiores e incontinência urinária e fecal. A utente foi referenciada para consulta externa de neurocirurgia, tendo sido detetada uma FAVDE.

Comentário: $O$ objetivo deste relato é evidenciar as dificuldades encontradas na avaliação da doente e aumentar a consciencialização para este problema raro, realçando a importância do diagnóstico e tratamento atempados.

Palavras-chave: Fístula arteriovenosa; Doenças vasculares da medula espinhal; Isquemia do cordão espinhal.

\section{INTRODUÇÃO}

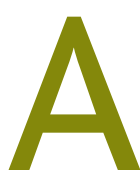

$\mathrm{s}$ fístulas arteriovenosas espinhais são raras e constituem cerca de $3 \%$ de todas as lesões da espinhal medula. ${ }^{1} \mathrm{O}$ subtipo dural é o mais frequente e representa $70-80 \%$ das malformações vasculares da espinhal medula. ${ }^{2-3}$ Apesar de a etiologia ser desconhecida, pensa-se que estas lesões sejam adquiridas. ${ }^{1,4-5}$

As fístulas arteriovenosas durais espinhais (FAVDE) tendem a localizar-se na região dorsal e lombar, sendo que $80 \%$ ocorrem no território T6-L2. ${ }^{3-4} \mathrm{O}$ shunt tem geralmente origem numa artéria radiculo-meníngea que drena para uma veia perimedular. ${ }^{1}$ Assim, sangue sob pressão arterial comunica diretamente com o plexo venoso, resultando na sua arterialização. ${ }^{3-5}$ Os fenómenos de congestão e hipertensão venosa desregulam o fluxo venoso medular, causando edema e isquemia e, consequentemente, mielopatia progressiva ascendente. ${ }^{1,3,5}$

O objetivo deste relato é evidenciar as dificuldades encontradas na avaliação da doente e aumentar a cons-

1. Médica Especialista em Medicina Geral e Familiar. USF Valbom, ACeS Gondomar. Gondomar, Portugal.

2. Médica Interna de Medicina Geral e Familiar. USF Sete Caminhos, ACeS Gondomar. Gondomar, Portugal.

2. Médica Especialista em Medicina Geral e Familiar. USF Sete Caminhos, ACeS Gondomar. Gondomar, Portugal. ciencialização para este problema raro, realçando a importância do seu reconhecimento e referenciação atempada pelo médico assistente.

\section{DESCRIÇÃO DO CASO}

Mulher de 50 anos, caucasiana, católica, com o ensino básico de escolaridade e doméstica de profissão. Integra uma família nuclear na fase VI do ciclo de $\mathrm{Du}$ vall. Apresenta hipertensão arterial medicada com amilorida $10 \mathrm{mg} i d$, diabetes mellitus tipo 2 controlada com alterações do estilo de vida, depressão reativa sob psicoterapia e asma tratada com corticoide inalado (budesonida $200 \mu \mathrm{g} i d$ ). Sem antecedentes familiares de relevo.

Em maio de 2019 dirigiu-se a uma consulta aberta no médico de família por queixas álgicas após queda acidental da própria altura - "tropeçou" (sic) - na semana anterior, com impacto na mão e anca direitas, joelhos bilateralmente e pé direito. Objetivou-se, do lado direito, dor à mobilização do $4 .^{\circ} \mathrm{e} 5 .^{\circ}$ dedos da mão, acrescida de edema ligeiro do joelho e pé. Foi prescrita analgesia (paracetamol 1000mg tid) e requisitados exames imagiológicos para exclusão de possível fratura (radiografia do punho, mão e tornozelo em carga direitos, bacia e joelhos em carga e anca), que não revelaram alterações de significado patológico. 
Em agosto do mesmo ano foi reavaliada por manutenção de queixas álgicas na anca direita com irradiação para a face posterior da coxa, de predomínio vespertino, e agravadas pelo movimento. Negou rigidez e febre. O exame objetivo denotou dor à mobilização ativa e passiva da anca direita e ausência de sinais inflamatórios. A manobra de Lasègue foi positiva à direita $\mathrm{e}$ a execução de marcha em pontas e calcanhar despoletou dor na anca direita. Tendo em conta o ritmo misto da dor, foi requisitado um estudo analítico com hemograma completo, proteína $C$ reativa e velocidade de sedimentação. Adicionalmente, realizou tomografia computorizada (TC) da coluna lombo-sagrada e anca direita para pesquisa de patologia osteoarticular inflamatória ou degenerativa. As imagens evidenciaram alterações degenerativas da coluna e entesopatia do grande trocânter direito. Iniciou terapêutica com pregabalina $75 \mathrm{mg}$ bid (com acréscimo da dose a cada quatro dias, se manutenção da dor), diclofenac tópico bid e tratamento de medicina física e de reabilitação.

Nos meses de setembro e outubro apresentou-se duas vezes na consulta aberta com agravamento da coxalgia, necessitando de apoio bilateral para a marcha, por dor e perceção de fraqueza muscular dos membros inferiores. Quando questionado o marido, este referiu que os sintomas aparentavam piorar nos fins-de-semana e período vespertino, particularmente nos momentos em que estava acompanhada pela família. Pelos antecedentes de patologia depressiva e interpretação como somatização foi medicada com sulpirida $50 \mathrm{mg} i d$, com ligeira melhoria das queixas.

Em dezembro de 2019, a doente foi observada por agravamento de sintomas com a presença de disestesias nos membros inferiores sem distribuição radicular, incontinência urinária de urgência e incontinência fecal (perda inconsciente de gases e fezes). Ao exame físico objetivou-se paraparésia dos membros inferiores e reflexos tendinosos rotuliano e aquiliano vivos. Perante estes achados foi ponderada a hipótese de compressão da espinhal medula e foi referenciada com urgência para consulta hospitalar de neurocirurgia.

Na consulta hospitalar, que se realizou em março de 2020, verificou-se ao exame objetivo paraparésia de predomínio esquerdo, disestesias ao toque no membro inferior direito e clónus bilateral dos pés, tendo sido colocada a hipótese diagnóstica de paraparésia esbo- çando síndroma de Brown-Séquard. Realizou ressonância magnética (RM), que demonstrou extenso hipersinal T2 e aparente expansão medular entre D3 e o cone medular, a que se associaram múltiplos flow voids perimedulares de aparência serpentiforme sugestivos de shunt arteriovenoso, com congestão venosa e edema do cordão medular. Posteriormente foi submetida a estudo angiográfico, que concluiu a presença de uma FAVDE no buraco de conjugação D11-D12 direito, constituída por um shunt entre uma artéria radiculo-meníngea de $\mathrm{D} 11$ direita e uma veia radículo-medular. O tratamento endovascular não foi possível; no entanto, a artéria aferente foi marcada para orientação cirúrgica. A exclusão cirúrgica da FAVDE decorreu sem intercorrências e no pós-operatório imediato verificou-se ligeira melhoria dos sintomas sensitivos e motores, embora sem capacidade para marcha autónoma. A doente encontra-se a realizar plano prolongado de reabilitação, em regime de internamento em unidade especializada.

\section{COMENTÁRIO}

\section{Epidemiologia e apresentação clínica}

As FAVDE afetam sobretudo o sexo masculino $(80 \%$ dos casos), em oposição ao caso descrito. ${ }^{4-5}$ Surgem maioritariamente na meia-idade, constituindo um diagnóstico extremamente incomum antes dos 30 anos. $^{3-6}$

Não existem características específicas na apresentação clínica das FAVDE e, geralmente, os sinais e sintomas têm uma evolução progressiva e insidiosa $\cdot^{3-5} \mathrm{Os}$ sintomas descritos como precoces são os distúrbios da marcha, como claudicação intermitente e paraparésia, e alterações sensitivas..$^{1-5}$ À semelhança deste caso, a maioria dos doentes apresenta uma combinação de sintomas (paresia, alterações sensitivas e disfunção de esfíncteres) no momento do diagnóstico. ${ }^{1,4}$

\section{Diagnóstico diferencial e exames complementares}

Atendendo à inespecificidade do quadro clínico e por se tratar de uma doença rara, muitos doentes são diagnosticados com outras patologias, como estenose espinhal, patologia degenerativa da coluna lombar, mielite inflamatória, neuropatia periférica, doença desmielinizante ou tumor medular. ${ }^{4-5,7}$ Em adição, dada a idade habitual de apresentação, muitos doentes 
apresentam achados clínicos e imagiológicos associados que dificultam o diagnóstico diferencial, por exemplo, deterioração dos discos intervertebrais, cirurgia prévia ou história de traumatismo. ${ }^{3}$ Estudos questionaram a possível etiologia traumática das FAVDE; no entanto, estima-se que apenas $4 \%$ dos casos apresentam antecedentes de trauma. ${ }^{4}$

Na perspetiva do médico de família, a primeira atitude tomada neste caso foi a exclusão de fratura no contexto de história de trauma. Tendo em conta a ocorrência da queda há uma semana e a ausência de achados alarmantes ao exame físico não se justificou observação em serviço de urgência. Perante a persistência das queixas foi iniciada investigação ao nível analítico e imagiológico, sendo o principal objetivo a exclusão de patologia osteoarticular degenerativa ou inflamatória de base, até agora desconhecida e eventualmente agravada pelo trauma. Perante os achados de doença degenerativa e entesopatia procedeu-se à analgesia adequada e tratamento de medicina física e reabilitação para reforço e alongamento da musculatura afetada. Atendendo à perspetiva psicológica e contexto familiar desta doente, os antecedentes de depressão reativa $\mathrm{e} o$ facto das queixas agravarem no momento em que se encontrava acompanhada pela família levantou a suspeita de sintomas somáticos. Assim, pensa-se que a presença de história prévia de trauma, os achados imagiológicos de doença osteoarticular degenerativa e os antecedentes de patologia depressiva contribuíram para o atraso do diagnóstico no caso descrito.

O agravamento súbito do quadro clínico e a presença de sinais de alarme, como a disfunção de esfíncteres, foi crucial na decisão do médico de família de referenciação da doente, permitindo o diagnóstico e intervenção terapêutica em menos de doze meses. Num departamento de neurologia espanhol, cerca de $60 \%$ dos doentes obtiveram um diagnóstico inicial errado. ${ }^{1}$ Em vários estudos é referido um atraso diagnóstico entre um a três anos desde o início da sintomatologia..$^{2-5,7}$

Os achados típicos na RM incluem edema centromedular em hipersinal T2, aumento do volume da espinhal medula e a presença de fluxo serpiginoso correspondente aos vasos perimedulares dilatados. ${ }^{1-5,7} \mathrm{O}$ estudo angiográfico seletivo permite a confirmação do diagnóstico, a marcação da artéria aferente e a avaliação do fluxo venoso. ${ }^{4-5}$

\section{Tratamento e prognóstico}

O objetivo do tratamento é ocluir a derivação sanguínea anómala e, para isso, é necessária uma cooperação entre neurorradiologia e neurocirurgia na definição do tratamento mais adequado a cada caso. ${ }^{4}$ Uma metaanálise não demonstrou qualquer vantagem da abordagem endovascular face ao tratamento cirúrgico e muitos doentes beneficiam de tratamento combinado. ${ }^{1,8}$

Nesta doente optou-se pelo tratamento cirúrgico, tendo este decorrido sem intercorrências. Não obstante, a doente não recuperou a capacidade de realizar marcha autónoma após a intervenção. Foi internada numa unidade de reabilitação; manteve-se, no entanto, sempre em estreita comunicação com o médico de família, tendo em vista a gestão das expectativas do doente e do próprio médico face a uma patologia raramente observada ao nível dos cuidados de saúde primários e a manutenção do plano de recuperação após a alta.

O prognóstico a longo prazo depende do tempo de evolução, da severidade da apresentação, da capacidade funcional prévia e da localização da fístula. Em adição, é consensual que a melhoria clínica mais importante se revela ao nível da afetação motora e da marcha ( $80 \%$ dos doentes), em contraste com as alterações sensitivas que sofrem recuperação em apenas um terço dos casos. ${ }^{4}$

Em conclusão, as FAVDE são raras e potencialmente tratáveis. O médico de família deve ponderar este diagnóstico diferencial em casos de claudicação ou mielopatia de causa desconhecida. A sua referenciação para neurocirurgia deve ser o mais precoce possível, já que um tratamento urgente tem impacto prognóstico.

\section{REFERÊNCIAS BIBLIOGRÁFICAS}

1. Ortega-Suero G, Porta Etessam J, Moreu Gamazo M, Rodríguez-Boto G. Spinal arteriovenous fistulas in adults: management of a series of patients treated at a neurology department. Neurologia. 2018;33(7): 438-48.

2. Derollez C, Tard C, Kazémi A, Vermersch P, Pruvo JP. A rare and treatable cause of medullar claudication: spinal dural arteriovenous fistula. Ochsner J. 2019;19(4):397-400.

3. Fox S, Hnenny L, Ahmed U, Meguro K, Kelly ME. Spinal dural arteriovenous fistula: a case series and review of imaging findings. Spinal Cord Ser Cases. 2017;3:17024.

4. Iglesias Gordo J, Martínez García R. Spinal dural arteriovenous fistulas: the most frequent vascular malformations of the spinal cord. Radiologia. 2018;60(3):237-49. 
5. Fugate JE, Lanzino G, Rabinstein AA. Clinical presentation and prognostic factors of spinal dural arteriovenous fistulas: an overview. Neurosurg Focus. 2012;32(5):E17.

6. Risavi BL, Sekula R, McQuone B, Radachy J.Type I AV fistula of the thoracic spinal cord. Am J Emerg Med. 2019;37(2):375.e5-6.

7. Jablawi F, Schubert GA, Dafotakis M, Pons-Kühnemann J, Hans FJ, Mull M. Long-term outcome of patients with spinal dural arteriovenous fistula: the dilemma of delayed diagnosis. AJNR Am J Neuroradiol. 2020;41(2):357-63.

8. Goyal A, Cesare J, Lu VM, Alvi MA, Kerezoudis P, Brinjikji W, et al. Outcomes following surgical versus endovascular treatment of spinal dural arteriovenous fistula: a systematic review and meta-analysis. J Neurol Neurosurg Psychiatry. 2019;90(10):1139-46.

\section{CONFLITO DE INTERESSES}

Os autores declaram não ter quaisquer conflitos de interesse.

\section{FINANCIAMENTO}

Os autores declaram que o trabalho relatado neste manuscrito não foi objeto de qualquer tipo de financiamento externo.

\author{
ENDEREÇO PARA CORRESPONDÊNCIA \\ Joana Rita Mendes \\ E-mail: joanaritamendes@gmail.com \\ https://orcid.org/0000-0003-0359-066X \\ Recebido em 23-06-2020 \\ Aceite para publicação em 03-07-2021
}

\section{ABSTRACT}

\section{MYELOPATHY OF RARE AND TREATABLE CAUSE: A CASE REPORT}

Introduction: Spinal dural arteriovenous fistulas (FAVDE) are rare and have an unspecific and insidious clinical presentation. Its etiology is unknown and the incidence is higher in middle-aged men.

Case Description: A 50-year-old woman, with a history of reactive depression, went to the family physician for pain after a fall with an impact on her right hip and knees. The physical exam and radiographic images did not show any pathological findings, however, the pain persisted despite the conservative treatment. Lower limb dysesthesia and urinary and fecal incontinence added to the initial symptoms of nocturne right hip pain. The patient was referred to a neurosurgery appointment and a FAVDE was detected.

Comment: The aim of this case report is to show the difficulties found in the evaluation of this patient and raise awareness for this rare pathology, enhancing the importance of a timely diagnosis and treatment.

Keywords: Arteriovenous fistula; Spinal cord vascular diseases; Spinal cord ischemia. 